\title{
Extended Sign Test by Ranks for Ordered Repeated Measure
}

\author{
Oyeka, I. C.A. Umeh, E. U. \\ Department Of Statistics, Nnamdi Azikiwe University, Awka, Nigeria \\ Email of the corresponding author: editus2002@yahoo.com
}

\begin{abstract}
This paper developed an alternative statistical method for the analysis of time or space ordered data. The proposed method used the ranks of successive differences between the observations adjust for the order, direction and magnitudes of the successive differences. Chi-squared test statistic based on the ranks of the successively ordered data is developed to test for the possible existence of any statistical differences between the scores by subjects in the time or space ordered population. Some sample data were used to illustrate the proposed method. The result of the analysis shows that the present method is as powerful as some of the existing nonparametric methods that can be used for the same purpose at least for the present data but preferably for use if the available data set is ordered in time or space.
\end{abstract}

Keywords: Sign test, ordered samples, ranks, time, and space.

\section{Introduction}

Sometimes a researcher may be interested in analyzing some data obtained from subjects at several points in time. These may include responses to diagnostic tests administered at several time periods; students' examination results during their years of study; candidates' scores at repeated job interviews; scores by soccer teams or competitors recorded for several seasons; etc. These types of data represent repeated measures or observations on each subject studied.

If a random sample of these repeated measures drawn from a number of related populations that do not satisfy the necessary assumptions for the use of parametric tests, then use of non-parametric method is indicated and preferable. Non parametric methods that readily suggest themselves for this purpose include the Cochran Q test which requires the observations to be dichotomous assuming only two possible values(Gibbons 1971, Gibbons 1993), Siegel, 1956; Hollander and Wolfe, 1999, Freidlin and Gastwirth 2000). the Extended Median Test for matched samples which analysis independent samples when the parametric t-test cannot used(Oyeka et al. 2010) and Ties Adjusted Extended Sign Test for Ordered Data which analyses ordered repeated measures that are related in time, space or condition, (Oyeka et al. 2012), etc. In this paper, we propose an alternative statistical method for the analysis of time ordered repeated measures. The proposed method is based on the extension of the sign test using the ranks of successive differences between the observations.

\section{The Proposed Method}

Let $\left(x_{i 1}, x_{i 2}, x_{i 3}, \ldots, x_{i k}\right)$ be the ith batch or block of observations or scores in a random sample of size " $\mathrm{n}$ " drawn from " $\mathrm{k}$ " related or time ordered populations $X_{1}, X_{2}, \ldots, X_{k}$ for $\mathrm{i}=1,2, \ldots, \mathrm{n}$ where $\mathrm{k}$ may be indexed in time. Populations, $X_{2}, \ldots, X_{k}$ may be measurements on as low as the ordinal scale.

To develop an extended sign test by ranks for the purpose, we let

$d_{i j}=x_{i j}-x_{i j+1}$

for $\mathrm{i}=1,2, \ldots, \mathrm{n} ; \mathrm{j}=1,2, \ldots, \mathrm{k}-1$

That is we take the differences between observations or scores on successive treatment levels or time period for each subject. Let

$u_{i j}=\left\{\begin{array}{l}1, \text { if } d_{i j}>0 \\ 0, \text { if } d_{i j}=0 \\ -1, \text { if } d_{i j}<0\end{array}\right.$

Thus $u_{i j}$ assume the value 1 , if $d_{i j}$ the difference between the scores earned at the $j$ th and $(j+1)$ th treatment levels, by the subject in the ith block is positive; the value 0 if it is zero; and the value -1 , if it is negative; if $i=1$, $2, \ldots, n ; j=1,2, \ldots, k$

Let

$\pi_{j}^{+}=P\left(u_{i j}=1\right) ; \pi_{j}^{0}=P\left(u_{i j}=0\right) ; \pi_{j}^{-}=P\left(u_{i j}=-1\right)$

where

$\pi_{j}^{+}+\pi_{j}^{0}+\pi_{j}^{-}=1$

To develop the extended sign test based on ranks, we now instead of using the difference $d_{i j}^{\prime} s$ directly themselves, we first ranks the absolute values of these differences within each batch or block either from the smallest to the largest or largest to the smallest. Tied absolute differences within each block are as usual assigned their mean ranks. 
Thus let $r_{i j}$ be the rank assigned to the absolute value of $d_{i j}$, the difference between observations from populations $X_{j}$ and $X_{j+1}$, in the ith block for $\mathrm{i}=1,2, \ldots, \mathrm{n} ; \mathrm{j}=1,2, \ldots, \mathrm{k}-1$.

Define

$W_{j}=\sum_{i=1}^{n} r_{i j} u_{i j}$

for $\mathrm{j}=1,2, \ldots, \mathrm{k}-1$

Also let

$\mathrm{W}=\sum_{j=1}^{k-1} W_{j}=\sum_{i=1}^{n} r_{i j} u_{i j}$

$W_{j}$ is the difference between the total or sum of the ranks assigned to absolute differences with positive signs and sum of the ranks assigned to absolute differences with negative signs between the scores by all subjects at treatment levels $X_{j}$ and $X_{j+1}$ for $\mathrm{j}=1,2, \ldots, \mathrm{k}-1$. That is $W_{j}$ measures the difference between the sum of the ranks assigned to positive differences and the sum of the ranks assigned to negative differences between observations from successive treatment levels or populations for all subjects. Note, $W_{j}$ is independent of zero differences. W is a measure of the overall total rank sum differences for all subjects and treatment levels. Interest is to use $\mathrm{W}$ as a test statistic for the null hypothesis of no difference between subjects in their response to the treatment levels. Now

$\mathrm{E}\left(u_{i j}\right)=\pi_{j}^{+}-\pi_{j}^{-} ; \operatorname{Var}\left(u_{i j}\right)=\pi_{j}^{+}+\pi_{j}^{-}-\left(\pi_{j}^{+}-\pi_{j}^{-}\right)^{2}$

The expected value of $W_{j}$ is from Equation 5

$\mathrm{E}\left(W_{j}\right)=\sum_{i=1}^{n} r_{i j} E\left(u_{i j}\right)=\left(\pi_{j}^{+}-\pi_{j}^{-}\right) \sum_{i=1}^{n} r_{i j}$

or

$\mathrm{E}\left(W_{j}\right)=\left(\pi_{j}^{+}-\pi_{j}^{-}\right) R_{. j}$

where

$R_{. j}=\sum_{i=1}^{n} r_{i j}$ is the sum of the ranks assigned to all the ' $\mathrm{n}$ ' absolute differences between the observations drawn from populations $X_{j}$ and $X_{j+1}, \mathrm{j}=1,2, \ldots, \mathrm{k}-1$.

Note that $\pi_{j}^{+} ; \pi_{j}^{0}$ and $\pi_{j}^{-}$are respectively the probabilities that observations from population $X_{j}$ are on the average greater than, equal to or less than observations from population $X_{j+1}$.

In other words, $\pi_{j}^{+} ; \pi_{j}^{0}$ and $\pi_{j}^{-}$provide a measure of the proportion of cases in which subjects scores are on the average rated or ranked higher (lower), the same as, or lower (higher) in treatment level or population $X_{j}$ than in treatment level $X_{j+1}$. The sample estimates of these probabilities are

$\hat{\pi}_{j}^{+}=\frac{f_{j}^{+}}{n} ; \hat{\pi}_{j}^{0}=\frac{f_{j}^{0}}{n} ; \hat{\pi}_{j}^{-}=\frac{f_{j}^{-}}{n}$

where $f_{j}^{+}, f_{j}^{0}$ and $f_{j}^{-}$are respectively the number of $1 \mathrm{~s}, 0 \mathrm{~s}$ and $-1 \mathrm{~s}$ in the frequency distribution of these numbers in $u_{i j}$, for $\mathrm{i}=1,2, \ldots, \mathrm{n}$ and each $\mathrm{j}=1,2, \ldots, \mathrm{k}-1$. Note that $f_{j}^{+}, f_{j}^{0}$ and $f_{j}^{-}$are respectively the number sampled subjects who are rated or ranked higher(lower), the same as, or lower(higher) at response or treatment level $X_{j}$ than at level $X_{j+1}$.

The variance of $w_{j}$ is obtained by using equation 7 in equation 5 giving

$\operatorname{Var}\left(w_{j}\right)=\sum_{i=1}^{n} r_{i j}^{2} \operatorname{Vr}\left(u_{i j}\right)=\left(\pi_{j}^{+}+\pi_{j}^{-}-\left(\pi_{j}^{+}-\pi_{j}^{-}\right)^{2}\right) \sum_{i=1}^{n} r_{i j}^{2}$

Note that $\pi_{j}^{+}-\pi_{j}^{-}$is the difference between the probabilities. That observation from population $X_{j}$ are on the average greater than observations from population $X_{j+1}$ and the probability that observations from population $X_{j}$ are on the average less than observations from population $X_{j+1}$. That is $\pi_{j}^{+}-\pi_{j}^{-}$provides a measure of the difference between the proportion of all subjects who on the average are rated or ranked higher at treatment level $X_{j}$ than at treatment level $X_{j+1}$ and the proportion of all subjects who are rated or ranked lower at treatment level $X_{j}$ than at treatment level $X_{j+1}$. This difference is estimated from the expression

$W_{j}=\left(\pi_{j}^{+}-\pi_{j}^{-}\right) R_{. j}=\widehat{W}_{j}^{+}-\widehat{W}_{j}^{-}$

where $\widehat{W}_{j}^{+}$and $\widehat{W}_{j}^{-}$are respectively the sum of the ranks of absolute differences with positive and negative signs of the differences between the scores of all subjects of treatment levels $X_{j}$ and $X_{j+1}$ for $\mathrm{j}=1,2, \ldots, \mathrm{k}-1$.

It can easily be seen from equations 8 and 11 that $W_{j}$ provide a measure of the amount by which the proportion of the total or sum of the ranks assigned to positive absolute differences between the scores of subjects at treatment levels $X_{j}$ and $X_{j+1}$ is on the average greater (lower) than the proportion of the total ranks assigned to negative absolute differences.

If populations $X_{j}$ and $X_{j+1}$ have equal medians, we would expect that $\pi_{j}^{+}-\pi_{j}^{-}$would be equal to zero for $\mathrm{j}=1$, $2, \ldots, \mathrm{k}-1$. In other words, a null hypothesis that would be of interest in this case is

$H_{o}: \pi_{j}^{+}-\pi_{j}^{-}=0$ versus $H_{1}: \pi_{j}^{+}-\pi_{j}^{-} \neq 0$, say for $\mathrm{j}=1,2, \ldots, \mathrm{k}-1$. 
Under this null hypothesis, the test statistic $\chi_{j}^{2}=\frac{w_{j}^{2}}{\operatorname{Var}\left(W_{j}\right)}$

for $\mathrm{j}=1,2, \ldots, \mathrm{k}-1$.

has approximately the Chi Square Distribution with degrees of freedom for significantly large 'n' and may be used to test the null hypothesis of equation 12 where $W_{j}$ and $\operatorname{Var}\left(W_{j}\right)$ are given in equations 11 and 10 respectively. $H_{o}$ is rejected at the $\alpha$ level of significant if

$\chi_{j}^{2} \geq \chi_{1-\alpha, 1}^{2}$

Otherwise $H_{o}$ is accepted.

However, to avoid committing a type II error too frequently, it is recommended that the calculated Chi-Square value of Equation 14 be compared with the tabulated Chi-Square value with k-1 degree of freedom instead of 1 degree of freedom.

However, a null hypothesis that is of greater interest here in the case of repeated measures is that $\mathrm{k}$ related populations have equal medians. To develop the required test statistic, we note from equation 6 that

$E(W)=\sum_{j=1}^{k-1} E\left(W_{j}\right)$ which using equation 8 yields

$E(W)=\sum_{j=1}^{k-1}\left(\pi_{j}^{+}-\pi_{j}^{-}\right) \sum_{i=1}^{n} r_{i j}=\sum_{j=1}^{k-1}\left(\pi_{j}^{+}-\pi_{j}^{-}\right) R_{. j}$.

Also from equation 10, we have

$\operatorname{Var}(W)=\sum_{j=1}^{k-1} \operatorname{Var}\left(W_{j}\right)=\sum_{j=1}^{k-1}\left(\pi_{j}^{+}+\pi_{j}^{-}-\left(\pi_{j}^{+}-\pi_{j}^{-}\right)^{2}\right) \sum_{i=1}^{n} r_{i j}^{2}$

Now it can be seen from Equation 15 that the sample estimate of $\sum_{j=1}^{k-1}\left(\pi_{j}^{+}-\pi_{j}^{-}\right) R_{. j}$ is given by the expression

$\mathrm{W}=\sum_{j=1}^{k-1}\left(\pi_{j}^{+}-\pi_{j}^{-}\right) R_{. j}=W^{+}-W^{-}$

where $W^{+}$and $W^{-}$are respectively the sums of the ranks of absolute differences with positive and negative signs of the differences between the scores of all subjects for all treatment levels.

Also note that $\sum_{j=1}^{k-1}\left(\pi_{j}^{+}-\pi_{j}^{-}\right) R_{. j}$ is a measure of how much on the average the proportion of the overall rank sums assigned to absolute differences with positive sign is greater (smaller) than the proportion of the overall rank sum of absolute differences with negative sign for all subjects and all sampled treatment levels.

Now if the k sampled populations have equal medians, that is if the differences between observations or scores in successive treatment levels are as likely to be positive or negative for all treatment levels, then the difference between $\pi_{j}^{+}$and $\pi_{j}^{-}$would be expected to be zero for all $\mathrm{j}=1,2, \ldots, \mathrm{k}-1$. In other words testing whether $\mathrm{k}$ related and here time ordered populations have equal medians is equivalent to testing the null hypothesis.

$H_{0}: \pi_{1}^{+}-\pi_{1}^{-}=\pi_{2}^{+}-\pi_{2}^{-}=\cdots=\pi_{k}^{+}-\pi_{k}^{-}=\pi^{+}-\pi^{-}=0$ versus $H_{1}$ : not all $\pi_{j}^{+}-\pi_{j}^{-}=0$

for some $j=1,2, \ldots, k-1$

where $\pi^{+}, \pi^{-}$and $\pi^{0}$ are respectively the common values of $\pi_{j}^{+}, \pi_{j}^{0}$ and $\pi_{j}^{-}$if $H_{o}$ is true for

$\mathrm{j}=1,2, \ldots, \mathrm{k}-1$.

The sample estimates of these common probabilities or proportions under the null hypothesis of equation 18 are respectively

$\hat{\pi}^{+}=\sum_{j=1}^{k-1} \frac{\hat{\pi}_{j}^{+}}{k-1}=\sum_{j=1}^{k-1} \frac{f_{j}^{+}}{n(k-1)}$,

$\hat{\pi}^{0}=\sum_{j=1}^{k-1} \frac{\widehat{\pi}_{j}^{0}}{k-1}=\sum_{j=1}^{k-1} \frac{f_{j}^{0}}{n(k-1)}$,

$\hat{\pi}^{-}=\sum_{j=1}^{k-1} \frac{\widehat{\pi}_{j}^{-}}{k-1}=\sum_{j=1}^{k-1} \frac{f_{j}^{-}}{n(k-1)}$

In this case, that is under $H_{o}$, the sample estimate of $\mathrm{W}$ (equation16) may be obtained from equation 17 as

$\mathrm{W}=\left(\pi_{j}^{+}-\pi_{j}^{-}\right) \sum_{j=1}^{k-1} R_{. j}=\left(\pi_{j}^{+}-\pi_{j}^{-}\right) R_{. .}=W^{+}-W^{-} \quad 20$

where

$R_{. .}=\sum_{j=1}^{k-1} R_{. j}=\frac{n k(k-1)}{2}$

and $W^{+}$and $W^{-}$are again respectively the sums of the ranks of absolute differences between scores with positive sign and those with negative sign for all subjects and all treatment levels.

The corresponding sample estimates of the variance of $\mathrm{W}$ under $H_{o}$ is from equation 16

$\operatorname{Var}(W)=\left(\hat{\pi}^{+}+\hat{\pi}^{-}-\left(\hat{\pi}^{+}-\hat{\pi}^{-}\right)^{2}\right) \sum_{j=1}^{k-1} \sum_{i=1}^{n} r_{i j}^{2}$

Note that if there are no tied observations in each of the ' $n$ ' blocks or batches of samples then the estimated variance of $\mathrm{W}$ (Equation 22) simplifies to

$\operatorname{Var}(W)=\frac{n k(k-1)(2 k-1)}{6}\left(\hat{\pi}^{+}+\hat{\pi}^{-}-\left(\hat{\pi}^{+}-\hat{\pi}^{-}\right)^{2}\right)$

Since under the situation $\sum_{j=1}^{k-1} \sum_{i=1}^{n} r_{i j}^{2}=\frac{n k(k-1)(2 k-1)}{6}$

Now under the null hypothesis of equation 18 , the test statistic 
$\chi^{2}=\frac{W_{j}^{2}}{\operatorname{Var}(W)}=\frac{W^{2}}{\left(\hat{\pi}^{+}+\hat{\pi}^{-}-\left(\hat{\pi}^{+}-\hat{\pi}^{-}\right)^{2}\right) \sum_{j=1}^{k-1} \sum_{i=1}^{n} r_{i j}^{2}}$

which under $H_{0}$ has approximately the Chi-Square Distribution with k-1 degree of freedom for significantly large ' $\mathrm{n}$ ' and may be used to test $H_{o}$ where $\mathrm{W}$ is given in Equation 20, the variance of $\mathrm{W}$ is given in Equation 22 if there are many tied absolute differences or by Equation 23 if there are no ties or only very few ties in the data.

The null hypothesis is rejected at the $\alpha$ level of significant if

$\chi^{2} \geq \chi_{1-\alpha, k-1}^{2}$

Otherwise, accepted. In this case, one would then proceed to test the null hypothesis of equation 12 to determine which pairs of successive populations or treatment levels have different medians that may have led to the rejection of the more general hypothesis of equation 18 .

\section{Illustrative Example}

Shown in Table 1 is the data on the grade point averages (GPA) of a random sample of 17 students during each of the four years they spent studying for a degree in an academic program of a certain university shown also in the Table are the successive differences $d_{i j}$ between three GPA's for each student by year.

Table 1: GPA's of a random sample of 17 students and their differences (Equation 1)

\begin{tabular}{|c|c|c|c|c|c|c|c|}
\hline S/No of Students & \multicolumn{4}{|l|}{ GPA } & \multicolumn{3}{|c|}{ Differences } \\
\hline & Year1 & Year2 & Year3 & Year4 & $d_{i 1}$ & $d_{i 2}$ & $d_{i 3}$ \\
\hline 1 & 3.7 & 1.7 & 2.2 & 4.0 & 2.0 & -0.5 & -1.8 \\
\hline 2 & 3.8 & 3.3 & 4.4 & 4.6 & 0.5 & -1.1 & -0.2 \\
\hline 3 & 4.1 & 4.0 & 4.4 & 4.3 & 0.1 & -0.4 & 0.1 \\
\hline 4 & 4.2 & 3.1 & 2.5 & 3.8 & 1.1 & 0.6 & -1.3 \\
\hline 5 & 3.7 & 3.3 & 4.3 & 4.3 & 0.4 & -1.0 & 0.0 \\
\hline 6 & 3.7 & 2.9 & 4.1 & 3.6 & 0.8 & -1.2 & 0.5 \\
\hline 7 & 2.8 & 2.1 & 3.1 & 3.3 & 0.7 & -1.0 & -0.2 \\
\hline 8 & 3.7 & 2.9 & 2.8 & 4.0 & 0.8 & 0.1 & -1.2 \\
\hline 9 & 4.1 & 2.7 & 4.0 & 3.9 & 1.4 & -1.3 & 0.1 \\
\hline 10 & 3.0 & 2.8 & 2.6 & 4.0 & 0.2 & 0.2 & -1.4 \\
\hline 11 & 3.5 & 2.5 & 3.7 & 3.7 & 1.0 & -1.2 & 0.0 \\
\hline 12 & 3.5 & 3.1 & 4.0 & 3.9 & 0.4 & -0.9 & 0.1 \\
\hline 13 & 4.5 & 4.4 & 4.6 & 4.7 & 0.1 & -0.2 & -0.1 \\
\hline 14 & 4.0 & 3.4 & 4.3 & 4.2 & 0.6 & -0.9 & 0.1 \\
\hline 15 & 3.8 & 3.5 & 3.9 & 4.0 & 0.3 & -0.4 & -0.1 \\
\hline 16 & 3.4 & 3.0 & 4.0 & 4.6 & 0.4 & -0.1 & -0.6 \\
\hline 17 & 3.9 & 4.0 & 4.4 & 4.7 & -0.1 & -0.4 & -0.3 \\
\hline
\end{tabular}

To illustrate use of the proposed method with the above data, we apply Equation 1 to the differences $d_{i j}$ in Table 1 to obtain the $U_{i j}{ }^{\prime} s$ of Equation 2. We also rank the differences in each block from the smallest to the largest, assigning the smallest absolute difference rank 1 and the largest rank3. Tied absolute differences in each block are assigned their mean ranks. The results are shown in Table 2.

Table 2: ranks of absolute values of $d_{i j}$ and values of $u_{i j}$ (Equation 2) for the data of Table 1

\begin{tabular}{|c|c|c|c|c|c|c|c|c|c|c|}
\hline S/No & $r_{i 1}$ & $u_{i 1}$ & $r_{i 1} u_{i 1}$ & $r_{i 2}$ & $u_{i 2}$ & $r_{i 2} u_{i 2}$ & $r_{i 3}$ & $u_{i 3}$ & $r_{i 3} u_{i 3}$ & Total \\
\hline 1 & 3 & 1 & 3 & 1 & -1 & -1 & 2 & -1 & -2 & \\
\hline 2 & 2 & 1 & 2 & 3 & -1 & -3 & 1 & -1 & -1 & \\
\hline 3 & 1.5 & 1 & 1.5 & 3 & -1 & -3 & 1.5 & 1 & 1.5 & \\
\hline 4 & 2 & 1 & 2 & 1 & 1 & 1 & 3 & -1 & -3 & \\
\hline 5 & 2 & 1 & 2 & 3 & -1 & -3 & 1 & 0 & 0 & \\
\hline 6 & 2 & 1 & 2 & 3 & -1 & -3 & 1 & 1 & 1 & \\
\hline 7 & 2 & 1 & 2 & 3 & -1 & -3 & 1 & -1 & -12 & \\
\hline 8 & 2 & 1 & 2 & 1 & 1 & 1 & 3 & -1 & -3 & \\
\hline 9 & 3 & 1 & 3 & 2 & -1 & -2 & 1 & 1 & 1 & \\
\hline 10 & 1.5 & 1 & 1.5 & 1.5 & 1 & 1.5 & 3 & -1 & -3 & \\
\hline 11 & 2 & 1 & 2 & 3 & -1 & -3 & 1 & 0 & 0 & \\
\hline 12 & 2 & 1 & 2 & 3 & -1 & -3 & 1 & 1 & 1 & \\
\hline 13 & 1.5 & 1 & 1.5 & 3 & -1 & -3 & 1.5 & -1 & -1.5 & \\
\hline 14 & 2 & 1 & 2 & 3 & -1 & -3 & 1 & 1 & 1 & \\
\hline 15 & 2 & 1 & 2 & 3 & -1 & -3 & 1 & -1 & -1 & \\
\hline 16 & 1 & 1 & 1 & 3 & -1 & -3 & 2 & -1 & -2 & \\
\hline 17 & 1 & -1 & -1 & 3 & -1 & -3 & 2 & -1 & -2 & \\
\hline$f_{j}^{+}$ & & 16 & & & 3 & & & 5 & & $24\left(=f^{+}\right)$ \\
\hline$f_{j}^{0}$ & & 0 & & & 0 & & & 2 & & $2\left(=f^{0}\right)$ \\
\hline$f_{j}^{-}$ & & 1 & & & 14 & & & 10 & & $25\left(=f^{-}\right)$ \\
\hline Total $\mathrm{n}$. & & 17 & & & 17 & & & 17 & & $51(n(k-1)$ \\
\hline$\hat{\pi}_{j}^{+}$ & & 0.941 & & & 0.176 & & & 0.294 & & $\left.0.471\left(\hat{\pi}^{+}\right)\right)$ \\
\hline$\hat{\pi}_{j}^{0}$ & & 0.00 & & & 0.00 & & & 0.118 & & $0.039\left(\hat{\pi}^{0}\right)$ \\
\hline$\hat{\pi}_{j}^{-}$ & & 0.059 & & & 0.824 & & & 0.588 & & $0.490\left(=\hat{\pi}^{-}\right)$ \\
\hline$R_{. j}$ & 32.5 & & & 42.5 & & & 27.0 & & & $102.0\left(=R_{\ldots}\right)$ \\
\hline$\frac{j}{W_{. j}}$ & & & 30.5 & & & -35.5 & & & -14.0 & $-19.0(=\mathrm{W})$ \\
\hline
\end{tabular}


The values of $f_{j}^{+}, f_{j}^{-}, f_{j}^{0}, \hat{\pi}_{j}^{+}, \hat{\pi}_{j}^{-}, \hat{\pi}_{j}^{0}, R_{. j}$ and $W_{. j}$ for $\mathrm{j}=1,2,3$ are calculated as discussed above and shown inTable2.

Now from equation 17 ,

$\mathrm{W}=(0.941-0.059)(32.5)+(0.176-0.824)(42.5)+(0.294-0.588)(27.0)$

$=(0.882)(32.5)+(0.648)(42.5)+(0.294)(27.0)$

$$
=28.665-27.54-7.938=6.813
$$

Also from equation 17 , we have that

$$
\begin{array}{r}
\operatorname{Var}(W)=\left(0.941+0.059-(0.941-0.059)^{2}(67.25)\right)+\left(0.176+0.824-(0.176-0.824)^{2}\right)(47.5) \\
+\left(0.294+0.588-(0.294-0.588)^{2}\right)(52.5)=14.930+689.5+41.79=124.87
\end{array}
$$

Henceforth equation 19 , we have that the test statistic for the equality of the four population medians is

$\chi^{2}=\frac{(-6.813)^{2}}{124.87}=0.372$ which with $4-1=3$ degrees of freedom is not statistically significant at $\alpha=$ $0.05\left(\chi_{0.95 ; 3}^{2}=7.815\right)$

Now the common values $\pi^{+}, \pi^{0}$ and $\pi^{-}$are estimated from equation 19 using the frequencies in Table 2 as $\hat{\pi}^{+}=\frac{24}{51}=0.471 ; \hat{\pi}^{0}=\frac{2}{51}=0.039 ;$ and $\hat{\pi}^{-}=\frac{25}{51}=0.490$

Also from Table 2, we have that $W=W_{1}+W_{2}+W_{3}=30.5-35.5-14.0=-19.0$

Since there are ties in the data, the variance of $W$ is estimated from Equation 22 as

$$
\begin{gathered}
\operatorname{Var}(W)=\left(0.471+0.490-(0.471-0.490)^{2}\right)(67.25+117.5+52.5) \\
=(0.9606)(237.25)=227.903
\end{gathered}
$$

Hence to test the null hypothesis of Equation 18 that the four population medians are equal, we have from Equation 24 that

$$
\chi^{2}=\frac{(-19.0)^{2}}{227.902}=\frac{361}{227.902}=1.584
$$

which with $4-1=3$ degrees of freedom is not statistically significant at

$$
\alpha=0.05\left(\chi_{0.95 ; 3}^{2}=7.815\right)
$$

Hence, we may conclude that the students did not experience differential academic performance during their four years of studies.

Note that the present test statistic is at least as powerful as the Wilcoxon Rank Sum Test. To show this, we note that relative efficiency of Wilcoxon $\left(t_{1}\right)$ to the new method $\left(t_{2}\right)$ is

\section{Summary and conclusion}

This paper developed an alternative statistical method for the analysis of time ordered repeated measures; from which an extension of the Sign Test using the ranks successive differences between the observations was proposed. From the result of the analysis, we may conclude that the students did not experience differential academic performance during their four years of studies.

\section{References}

Freidlin , B. and Gastwirth, J. L. (1987): Should the Median Test be Retired from General Use? American Statistician, 54, 161-164

Gibbons, J. D. (1971): Non- Parametric Statistical Inference. McGraw Hill, New York.

Gibbons, J. D.: Non- Parametric Statistical. An Introduction; Newbury Park: Sage Publication 1993

Hollander, M. and Wolfe, D.A. (1999): Non-Parametric Statistical Methods ( ${ }^{\text {nd }}$ Edition). Wiley InterScience, New York.

Oyeka, C. A., Ebuh, G.U., Nwankwo, C.C., Obiora- Ilouno, H. Ibeakuzie, P. O., Utazi, C. (2010): A Statistical Comparison of Test Scores: A Non- Parametric Approach. Journal of Mathematical Sciences. Vol 21. No 1. Pp77-87

Siegel, S. (1956): Non- Parametric Statistics for the Behavioural Sciences. McGraw- Hill, Kogakusha, Ltd, Tokyo. 
The IISTE is a pioneer in the Open-Access hosting service and academic event management. The aim of the firm is Accelerating Global Knowledge Sharing.

More information about the firm can be found on the homepage:

http://www.iiste.org

\section{CALL FOR JOURNAL PAPERS}

There are more than 30 peer-reviewed academic journals hosted under the hosting platform.

Prospective authors of journals can find the submission instruction on the following page: http://www.iiste.org/journals/ All the journals articles are available online to the readers all over the world without financial, legal, or technical barriers other than those inseparable from gaining access to the internet itself. Paper version of the journals is also available upon request of readers and authors.

\section{MORE RESOURCES}

Book publication information: http://www.iiste.org/book/

Academic conference: http://www.iiste.org/conference/upcoming-conferences-call-for-paper/

\section{IISTE Knowledge Sharing Partners}

EBSCO, Index Copernicus, Ulrich's Periodicals Directory, JournalTOCS, PKP Open Archives Harvester, Bielefeld Academic Search Engine, Elektronische Zeitschriftenbibliothek EZB, Open J-Gate, OCLC WorldCat, Universe Digtial Library, NewJour, Google Scholar

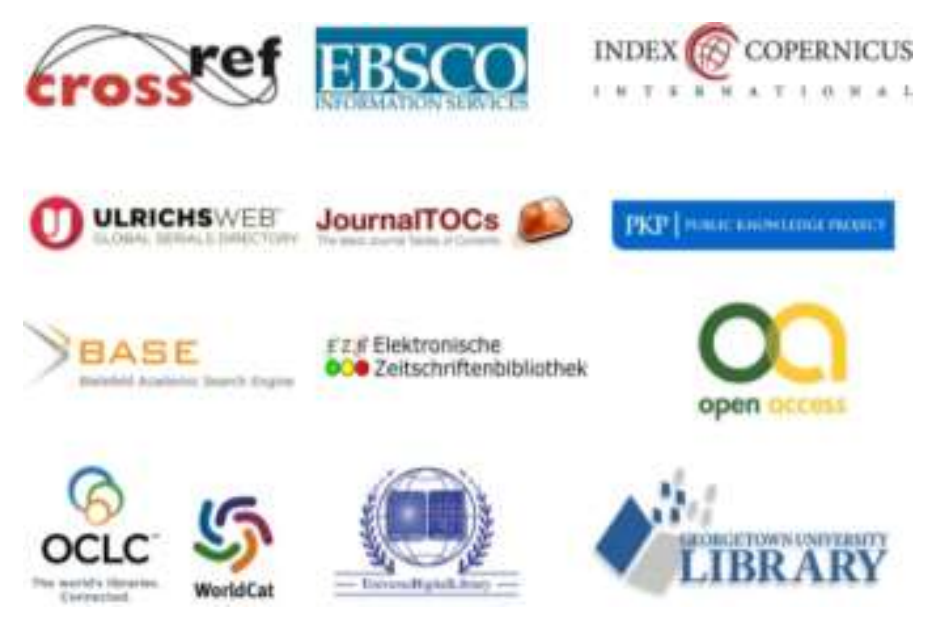

